\title{
Mutational Analysis for Wilson's Disease
}

WK Seto (MRCP) $)^{a}, C M$ Mak $(P h D)^{b}$, D But $(M R C P)^{a}$, I Hung $(M R C P)^{a}, C W$ Lam $(P h D)^{c}$, S Tam (FRCP) ${ }^{\mathrm{d}}$, MF Yuen (FRCPE) ${ }^{\mathrm{e}}, \mathrm{CL}$ Lai (FRCP)

${ }^{\mathrm{a}}$ Department of Medicine, the University of Hong Kong, Queen Mary Hospital

${ }^{b}$ Department of Pathology, Princess Margaret Hospital

'Professor, Department of Pathology, the University of Hong Kong, Queen Mary Hospital

${ }^{d}$ Department of Pathology, the University of Hong Kong, Queen Mary Hospital

${ }^{\mathrm{e}}$ Professor, Department of Medicine, the University of Hong Kong, Queen Mary Hospital

WK Seto and CM Mak are first co-authors of this case report

\section{Case}

We present a 30-year-old man with chronic hepatitis $B(\mathrm{CHB})$ infection. On referral in March 2005, he had no symptoms. He was hepatitis B e antigen (HBeAg) positive, had an alanine aminotransferase (ALT) of $182 \mathrm{U} / \mathrm{L}$. and a HBV-DNA of $6.68 \times 10^{5} \mathrm{IU} / \mathrm{ml}$. He was started on lamivudine $100 \mathrm{mg}$ daily, which was the only available nucleoside analogue at that time. His ALT and HBV-DNA remained elevated after 18 months of lamivudine and adefovir 10mg daily was subsequently added. However after 12 months of combination therapy, his ALT remained persistently elevated at $160 \mathrm{U} / \mathrm{L}$, though his HBV-DNA had dropped to $85.9 \mathrm{IU} / \mathrm{ml}$.

Other investigations for causes for chronic hepatitis were unremarkable, except for his serum ceruloplasmin level which was noted twice at $30 \mathrm{mg} / \mathrm{L}$ (normal 180-350 mg/L). Twentyfour hour urine copper was elevated to $2.26 \mu \mathrm{mol} /$ day (normal $<0.5 \mu \mathrm{mol} / \mathrm{day}$ ). He had no clinical features of extrapyramidal involvement. Slit-lamp examination also showed no KayserFleischer rings. Mutational analysis of the Wilson Disease (WD)-causing gene (ATP7B) by DNA sequencing detected two compound heterozygous disease-causing mutations: ATP7B 
NM_000053.2: c.2604delC (p.Pro868fs) and p.ASP1047Val (Figure 1). His parents were carriers of p.Pro868fs and p.Asp1047Val respectively. With the diagnosis of WD confirmed, he was started on penicillamine in October 2007, and his ALT normalized 6 months later. His ALT was $23 \mathrm{U} / \mathrm{L}$ on his most recent follow-up in March 2009.

\section{Discussion}

WD, first described by the American neurologist Samuel Alexander Kinnier Wilson in 1912, is inherited in an autosomal recessive disorder of copper metabolism which is characterized mainly by neurological manifestations and liver cirrhosis. It is traditionally perceived as a rare disorder, with a quoted prevalence of 1 in 30,000, and incidence ranging from 15 to 30 per million ${ }^{1}$. These figures were based on epidemiological data from the 1960 s to 1980s, and thus might lead to underestimation of the true prevalence of the disease. More sensitive diagnostic technology has shown a higher prevalence of WD, as common as 1 in 3,667 in East Asia ${ }^{2}$.

Conventionally, the diagnosis of WD is based on at least two of the following: the presence of Kayser-Fleischer rings, typical neurological symptoms, and a low serum ceruloplasmin level. However, WD can present with a wide range of clinical features, with the fulfillment of the above clinical criteria is often limited to patients with full-blown presentations. Diagnosing WD requires a high index of suspicion; the mean delay from symptoms to diagnosis has been reported to be 2 years (range: $0.08-30$ years) $)^{3}$. Our patient had no symptoms and only presented with an elevated ALT, despite the lowering of his HBV-DNA. Quantitation of 
hepatic copper by liver biopsy can be very useful, with hepatic copper $>250 \mu \mathrm{g} / \mathrm{g}$ dry weight providing a sensitivity of $83.3 \%$ and a specificity of $98.6 \%$, while values $<75 \mu \mathrm{g} / \mathrm{g}$ exclude the diagnosis ${ }^{4}$. However, levels between 75 and $250 \mu \mathrm{g} / \mathrm{g}$ are inconclusive. Moreover, liver biopsy is an invasive procedure.

In our experience, genetic analysis of $A T P 7 B$ remains the most decisive and non-invasive diagnostic tool, with a mutation detection rate of $97.6 \%^{5}$ The two disease-causing mutations of our patient p.Pro868fs and p.Asp1047val, are known mutations found in Hong Kong Chinese WD patients.

Since both CHB and WD are common in East Asian populations, one should be vigilant for other causes of elevated ALT levels in patients with known CHB. While traditional biochemical markers like serum ceruloplasmin and 24-hour urine copper should always be used as first-line investigations, they should be supplemented with molecular genetic studies in case of diagnostic difficulties. With WD more common than traditionally perceived, mutational analysis has the potential to be applied at a broader basis before symptoms occur.

(Word count: 600)

The authors declare they have participated in the preparation of the case report and have seen and approved the final version. 


\section{Conflicts of Interest}

All authors have no conflicts of interest

Role of the funding source

All authors have nothing to disclose 


\section{References}

1. Scheinberg I, Sternlieb I. Wilson's Disease. Major Probl Intern Med 1984; 23: 1-24.

2. Hahn SH, Lee SY, Jang SJ, et al. Pilot study of mass screening for Wilson's disease in Korea. Mol Genet Metab 2002; 76: 133-136.

3. Prashanth LK, Taly AB, Sinha S, et al. Wilson's disease: diagnostic errors and clinical implications. J Neurol Neurosurg Psychiatry 2004; 75: 907-909.

4. Ferenci $P$, Steindl-Munda $P$, Vogel $W$, et al. Diagnostic value of quantitative hepatic copper determination in patients with Wilson's Disease. Clin Gastroenterol Hepatol 2005; 3: 811-818.

5. Mak CM, Lam CW, Tam S, et al. Mutational analysis of 65 Wilson disease patients in Hong Kong Chinese - Identification of 17 novel mutations and its genetic heterogeneity. J Hum Genet 2008; 53: 55-63. 


\section{Figure 1}

The DNA sequences in sense direction of the proband show (a) ATP7B NM 000053.2:c.2604delC (p.Pro868ProfsX\#5) and (b) p.Asp1047Val respectively (indicated by arrow).

(a) A A A C C N G N A G N

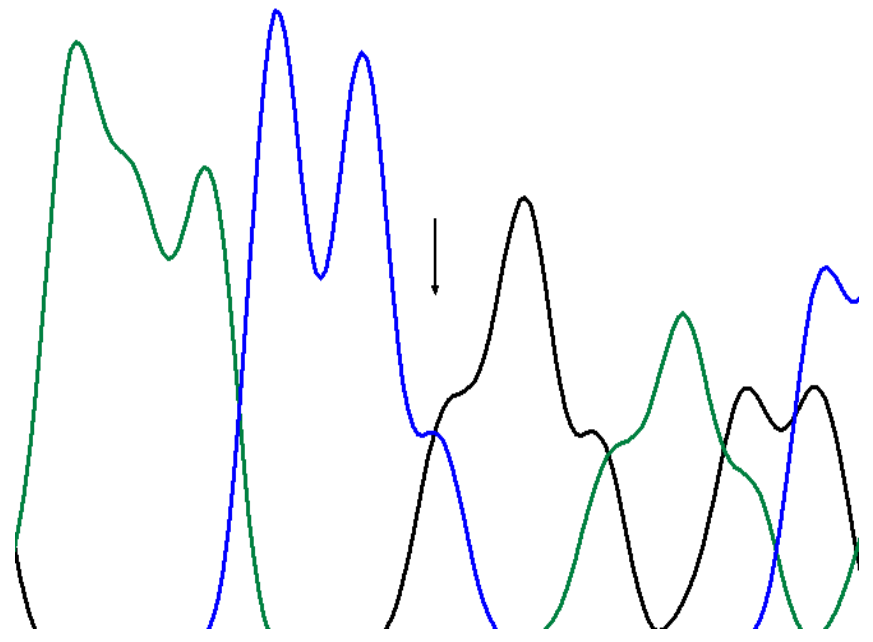

(b) G G G N T G T G

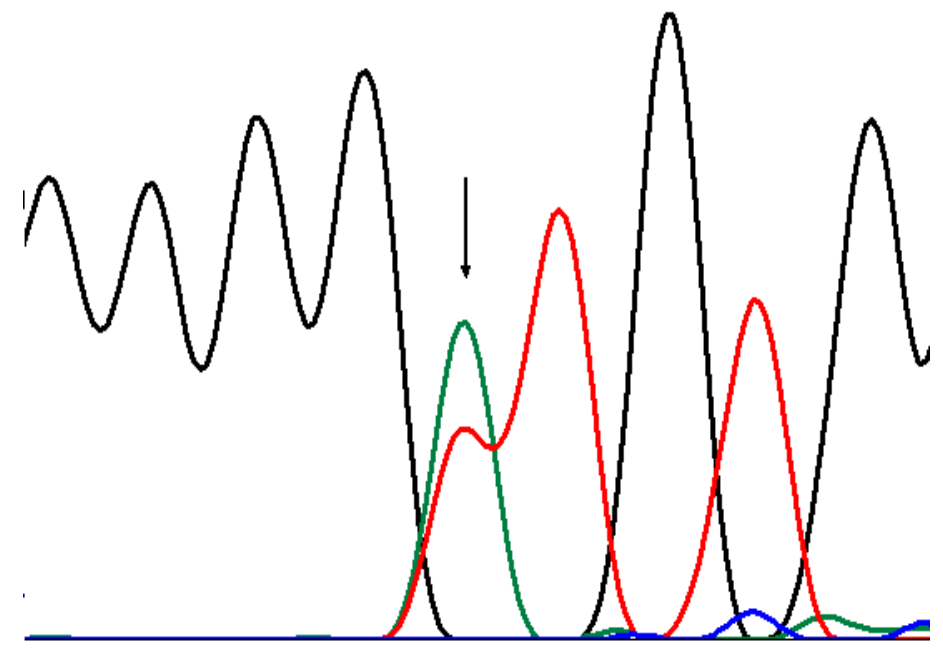

